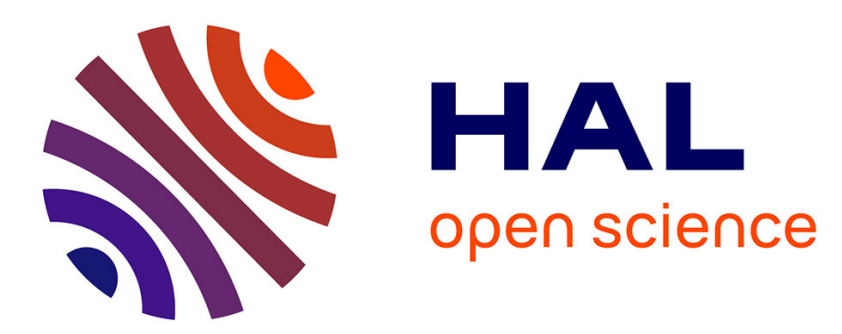

\title{
Global and Local Weighted Homogeneity for Time-Delay Systems
}

\author{
Denis Efimov, Wilfrid Perruquetti, Jean-Pierre Richard
}

\section{To cite this version:}

Denis Efimov, Wilfrid Perruquetti, Jean-Pierre Richard. Global and Local Weighted Homogeneity for Time-Delay Systems. Karafyllis I.; Malisoff M.; Mazenc F.; Pepe P. Recent Results on Nonlinear Delay Control Systems, 4, Springer, pp.163-181, 2015, 978-3-319-18071-7. hal-01185726

\section{HAL Id: hal-01185726 \\ https://hal.inria.fr/hal-01185726}

Submitted on 21 Aug 2015

HAL is a multi-disciplinary open access archive for the deposit and dissemination of scientific research documents, whether they are published or not. The documents may come from teaching and research institutions in France or abroad, or from public or private research centers.
L'archive ouverte pluridisciplinaire HAL, est destinée au dépôt et à la diffusion de documents scientifiques de niveau recherche, publiés ou non, émanant des établissements d'enseignement et de recherche français ou étrangers, des laboratoires publics ou privés. 


\title{
Global and Local Weighted Homogeneity for Time-Delay Systems
}

\author{
Efimov Denis, Perruquetti Wilfrid and Richard Jean-Pierre
}

\begin{abstract}
The notion of weighted homogeneity is extended to the time-delay systems. It is shown that the stability/instability of homogeneous functional systems on a sphere implies the global stability/instability of the system. The notion of local homogeneity is introduced, a relation between stability/instability of the locally approximating dynamics and the original time-delay system is established using Lyapunov-Razumikhin approach. An implication between homogeneity and inputto-state stability is investigated. Examples of application of the proposed theory are given.
\end{abstract}

\section{Introduction}

For homogeneous ordinary differential equations (ODEs), the global behavior of trajectories can be evaluated based on their behavior on a suitably defined sphere around the origin [36]. Thus the local and global behaviors of homogeneous systems are the same. This property has been found useful for stability analysis $[2,4,25,27$, $35]$, approximation of system dynamics [24,30], stabilization [7, 18, 28,32,38] and

Efimov Denis

Non-A team @ Inria, 40 avenue Halley, 59650 Villeneuve d'Ascq, France and Department of Control Systems and Informatics, Saint Petersburg State University of Information Technologies Mechanics and Optics (ITMO), Kronverkskiy av. 49, Saint Petersburg, 197101, Russia, e-mail: Denis.Efimoveinria.fr

Perruquetti Wilfrid

Non-A team @ Inria, 40 avenue Halley, 59650 Villeneuve d'Ascq, France and LAGIS (UMRCNRS 8146), Ecole Centrale de Lille, 59651 Villeneuve-d'Ascq, France e-mail: wilfrid. perruquettieinria.fr

Richard Jean-Pierre

Name, Non-A team @ Inria, 40 avenue Halley, 59650 Villeneuve d'Ascq, France and LAGIS (UMR-CNRS 8146), Ecole Centrale de Lille, 59651 Villeneuve-d'Ascq, France e-mail: jean-pierre.richardeec-lille.fr 
estimation [2,30]. It has been shown that for stability/instability analysis, Lyapunov function of a homogeneous system can be chosen homogeneous $[13,35,40]$. Thus the numerical analysis and design of homogeneous systems may be simpler since, for example, a Lyapunov function has to be constructed on a sphere only (on the whole state space it can extended using homogeneity).

Definitely, the class of nonlinear systems with a globally (stable/unstable) behavior is rather restricted. That is why the notion of local homogeneity has been defined and widely used $[2,13,40]$. This concept implies that the system is similar to a homogeneous one on a sphere only. In this case the theory of homogeneous systems can be applied to analyze locally (around that sphere) the behavior of the original nonlinear system.

The theory of homogeneous systems has been developed for time-invariant ODEs. Another important class of systems can be modeled by the functional differential equations (FDEs), and in particular the systems with time delays. The delay presence is typical in many application domains $[9,16,34]$. For example, the networked systems suffer from various sources of delays (such as access time, communication or packet dropouts), as well as the embedded/real-time systems (the asynchronous sampling effect can be modeled by delays, see [17] and the references herein). Appearance of a delay impacts critically the system stability and behavior complexity $[19,23,29]$, that is why most of the examples in the literature are modeled by linear time-delay systems. As a matter of fact, the analysis for such models, which is well developed (even the converse Lyapunov-Krasovskii theorems in the case of constant delays have been proposed [19]). For nonlinear time-delay systems, design of a Lyapunov-Krasovskii functional or a Lyapunov-Razumikhin function is a difficult problem. As it has been shown in the ODE case, homogeneity may simplify analysis and design of nonlinear systems, and this is a motivation for development of the homogeneity theory to FDEs.

The main obstruction for such a development is that the time-delay systems operate in the infinite dimensional state space. An extension of the standard results $[2,13,35,40]$ to this class of systems needs a complete revision of the arguments and tools of the theory of homogeneous system developed for ODEs. There exist a few works, which deal with time-delay systems using the homogeneity theory $[1,3,8,11]$. In all these works the homogeneity theory for ODEs has been applied to the delay-free part of the systems, and the concept of homogeneity has not been extended to the functional spaces. In the recent works $[14,15]$, the homogeneity theory has been extended to FDEs: the present chapter mainly follows these works, and some new results and examples are considered.

The outline of this chapter is as follows. The preliminary definitions and the system equations are given in Section 2. The homogeneous norm, an extended definition of homogeneity for time-delay systems and sufficient stability/instability conditions are presented in Section 3. The local homogeneity theory is studied in Section 4. In $[5,26,37]$ it has been shown that for ODEs the homogeneity implies some kind of robustness with respect to external disturbances, a corresponding link between input-to-state stability (ISS) and homogeneity for FDEs is established in Section 5. Examples are presented in Section 6. 


\section{Preliminaries}

Consider an autonomous functional differential equation of the retarded type [29]:

$$
d x(t) / d t=f\left(x_{t}\right), t \geq 0
$$

where $x \in \mathbb{R}^{n}$ and $x_{t} \in C_{[-\tau, 0]}$ is the state function, $x_{t}(s)=x(t+s),-\tau \leq s \leq 0$ (we denote by $C_{[-\tau, 0]}, 0<\tau<+\infty$ the Banach space of continuous functions $\phi:[-\tau, 0] \rightarrow \mathbb{R}^{n}$ with the uniform norm $\|\phi\|=\sup _{-\tau<\varsigma<0}|\phi(\varsigma)|$, where $|\cdot|$ is the standard Euclidean norm); $f: C_{[-\tau, 0]} \rightarrow \mathbb{R}^{n}$ is a locally Lipschitz continuous function, $f(0)=0$. The representation (1) includes pointwise or distributed retarded systems with either constant or variable time delay $\tau(t) \in[0, \tau]$. We assume that solutions of the system (1) satisfy the initial functional condition $x_{0} \in C_{[-\tau, 0]}$. It is known from the theory of functional differential equations [29] that under the above assumptions the system (1) has a unique solution $x\left(t, x_{0}\right)$ satisfying the initial condition $x_{0}$, which is defined on some finite time interval $[-\tau, T$ ) (we will use the notation $x(t)$ to reference $x\left(t, x_{0}\right)$ if the origin of $x_{0}$ is clear from the context).

For a locally Lipschitz continuous function $V: \mathbb{R}^{n} \rightarrow \mathbb{R}_{+}$the lower or upper directional Dini derivatives are defined as follows:

$$
\begin{aligned}
D^{-} V\left[x_{t}(0)\right] f\left(x_{t}\right) & =\lim _{h \rightarrow 0^{+}} \inf \frac{V\left[x_{t}(0)+h f\left(x_{t}\right)\right]-V\left[x_{t}(0)\right]}{h} \\
D^{+} V\left[x_{t}(0)\right] f\left(x_{t}\right) & =\lim _{h \rightarrow 0^{+}} \sup \frac{V\left[x_{t}(0)+h f\left(x_{t}\right)\right]-V\left[x_{t}(0)\right]}{h} .
\end{aligned}
$$

A continuous function $\sigma: \mathbb{R}_{+} \rightarrow \mathbb{R}_{+}$belongs to class $\mathcal{K}$ if it is strictly increasing and $\sigma(0)=0$; it belongs to class $\mathcal{K}_{\infty}$ if it is also radially unbounded. A continuous function $\beta: \mathbb{R}_{+} \times \mathbb{R}_{+} \rightarrow \mathbb{R}_{+}$belongs to class $\mathcal{K} \mathcal{L}$ if $\beta(\cdot, r) \in \mathcal{K}$ and $\beta(r, \cdot)$ is strictly decreasing to zero for any fixed $r \in \mathbb{R}_{+}$. The symbol $\overline{1, m}$ denotes a sequence of integers $1, \ldots, m$.

\section{Homogeneity}

For any $r_{i}>0, i=\overline{1, n}$ and $\lambda>0$, define the dilation linear operator $\Lambda_{r}(\lambda)=$ $\operatorname{diag}\left\{\lambda^{r_{i}}\right\}_{i=1}^{n}$ and the vector of weights $r=\left[r_{1}, \ldots, r_{n}\right]^{T}$.

For any $r_{i}>0, i=\overline{1, n}$ and $x \in \mathbb{R}^{n}$ the homogeneous norm can be defined as follows

$$
|x|_{r}=\left(\sum_{i=1}^{n}\left|x_{i}\right|^{\rho / r_{i}}\right)^{1 / \rho}, \rho=\prod_{i=1}^{n} r_{i} .
$$

For all $x \in \mathbb{R}^{n}$, the Euclidean norm $|x|$ is related with the homogeneous one: 


$$
\begin{gathered}
\underline{\sigma}_{r}\left(|x|_{r}\right) \leq|x| \leq \bar{\sigma}_{r}\left(|x|_{r}\right), \\
\bar{\sigma}_{r}(s)=\max _{|x|_{r} \leq s}|x|, \underline{\sigma}_{r}^{-1}(s)=\max _{|x| \leq s}|x|_{r},
\end{gathered}
$$

where $\underline{\sigma}_{r}, \bar{\sigma}_{r} \in \mathcal{K}_{\infty}$ define the Euclidean norm deviations with respect to the homogeneous norm. The homogeneous norm has an important property that $\left|\Lambda_{r}(\lambda) x\right|_{r}=$ $\lambda|x|_{r}$ for all $x \in \mathbb{R}^{n}$. Define $\mathbb{S}_{r}=\left\{x \in \mathbb{R}^{n}:|x|_{r}=1\right\}$.

Since the transformation by dilation operator $\Lambda_{r}(\lambda)$ is linear, it can be applied to functional arguments. Indeed, for any $r_{i}>0, i=\overline{1, n}$ and $\phi \in C_{[a, b]}, 0 \leq a<b \leq$ $+\infty$ the homogeneous norm can be defined as follows:

$$
\|\phi\|_{r}=\left(\sum_{i=1}^{n}\left\|\phi_{i}\right\|^{\rho / r_{i}}\right)^{1 / \rho}, \rho=\prod_{i=1}^{n} r_{i} .
$$

Lemma 1. [15] There exist two functions $\underline{\rho}_{r}, \bar{\rho}_{r} \in \mathcal{K}_{\infty}$ such that for all $\phi \in C_{[a, b]}$

$$
\underline{\rho}_{r}\left(\|\phi\|_{r}\right) \leq\|\phi\| \leq \bar{\rho}_{r}\left(\|\phi\|_{r}\right) .
$$

Therefore, the proposed homogeneous norm is equivalent to the uniform norm in $C_{[a, b]}$. The homogeneous norm in the Banach space has the same important property that $\left\|\Lambda_{r}(\lambda) \phi\right\|_{r}=\lambda\|\phi\|_{r}$ for all $\phi \in C_{[a, b]}$. Define the corresponding unit sphere $\mathcal{S}_{r}=\left\{\phi \in C_{[-\tau, 0]}:\|\phi\|_{r}=1\right\}$.

Definition 1. The function $g: C_{[-\tau, 0]} \rightarrow \mathbb{R}$ is called $r$-homogeneous $\left(r_{i}>0\right.$, $i=\overline{1, n})$, if for any $\phi \in C_{[-\tau, 0]}$ the relation

$$
\lambda^{-d} g\left(\Lambda_{r}(\lambda) \phi\right)=g(\phi)
$$

holds for some $d \in \mathbb{R}$ and all $\lambda>0$.

The function $f: C_{[-\tau, 0]} \rightarrow \mathbb{R}^{n}$ is called $r$-homogeneous $\left(r_{i}>0, i=\overline{1, n}\right)$, if for any $\phi \in C_{[-\tau, 0]}$ the relation

$$
\lambda^{-d} \Lambda_{r}^{-1}(\lambda) f\left(\Lambda_{r}(\lambda) \phi\right)=f(\phi)
$$

holds for some $d \geq-\min _{1 \leq i \leq n} r_{i}$ and all $\lambda>0$.

In both cases, the constant $d$ is called the degree of homogeneity.

The introduced notion of homogeneity in $C_{[-\tau, 0]}$ is reduced to the standard one in $\mathbb{R}^{n}$ [35] under a vector argument substitution. An advantage of homogeneous systems described by nonlinear ordinary differential equations is that analysis of their stability can be performed on the unit sphere $\mathbb{S}_{r}$ only [35] (the homogeneous system trajectories have a similar behavior on any other sphere defined by the norm $\left.|\cdot|_{r}\right)$. This conclusion is based on the property that any solution of a homogeneous system can be obtained from another solution under the dilation rescaling and a suitable time re-parametrization. A similar property holds for functional homogeneous systems. 
Proposition 1. Let (1) be the r-homogeneous system with the degree $d$ and $x\left(t, x_{0}\right)$ be its solution for an initial condition $x_{0} \in C_{[-\tau, 0]}, \tau \in(0,+\infty)$. For any $\lambda>0$ the functional differential equation

$$
d y(t) / d t=f\left(y_{t}\right), t \geq 0
$$

with $y_{t} \in C_{\left[-\lambda^{-d} \tau, 0\right]}$, has a solution $y\left(t, y_{0}\right)=\Lambda_{r}(\lambda) x\left(\lambda^{d} t, x_{0}\right)$ for all $t \geq 0$ with the initial condition $y_{0} \in C_{\left[-\lambda^{-d} \tau, 0\right]}, y_{0}(s)=\Lambda_{r}(\lambda) x_{0}\left(\lambda^{d} s\right)$ for $s \in\left[-\lambda^{-d} \tau, 0\right]$.

Proof. By definition $x_{\lambda^{d} t}(s)=x\left(\lambda^{d} t+s\right) \in \mathbb{R}^{n}$ and $y_{t}(s)=\Lambda_{r}(\lambda) x_{\lambda^{d} t}(s)$ for any $-\lambda^{-d} \tau \leq s \leq 0$, then

$$
\dot{y}(t)=\frac{d}{d t}\left(\Lambda_{r}(\lambda) x\left(\lambda^{d} t\right)\right)=\lambda^{d} \Lambda_{r}(\lambda) f\left(x_{\lambda^{d} t}\right)=f\left(\Lambda_{r}(\lambda) x_{\lambda^{d} t}\right)=f\left(y_{t}\right)
$$

and $y(t)$ is a solution of (2).

In order to better explain this result consider a particular case of the system (1) with the pointwise delay:

$$
f\left(x_{\tau}\right)=F[x(t), x(t-\tau)],
$$

then the homogeneity condition reads as $F\left[\Lambda_{r}(\lambda) x, \Lambda_{r}(\lambda) z\right]=\lambda^{d} \Lambda_{r}(\lambda) F[x, z]$ for any $x, z \in \mathbb{R}^{n}$ and $\lambda \in(0,+\infty)$. Select a solution $x\left(t, x_{0}\right)$ of this system for the initial condition $x_{0} \in C_{[-\tau, 0]}$ for some $\tau>0$ as before (i.e. $\dot{x}\left(t, x_{0}\right)=$ $\left.F\left[x\left(t, x_{0}\right), x\left(t-\tau, x_{0}\right)\right]\right)$. Take some $\lambda \in(0,+\infty)$ and define $y_{0} \in C_{\left[-\lambda^{-d} \tau, 0\right]}$, $y_{0}(s)=\Lambda_{r}(\lambda) x_{0}\left(\lambda^{d} s\right)$ for $s \in\left[-\lambda^{-d} \tau, 0\right]$, then consider $y\left(t, y_{0}\right)=\Lambda_{r}(\lambda) x\left(\lambda^{d} t, x_{0}\right)$, taking its derivative with respect to time we obtain

$$
\begin{aligned}
\frac{d y\left(t, y_{0}\right)}{d t} & =\frac{d \Lambda_{r}(\lambda) x\left(\lambda^{d} t, x_{0}\right)}{d t} \\
& =\lambda^{d} \Lambda_{r}(\lambda) F\left[x\left(\lambda^{d} t, x_{0}\right), x\left(\lambda^{d} t-\tau, x_{0}\right)\right] \\
& =F\left[\Lambda_{r}(\lambda) x\left(\lambda^{d} t, x_{0}\right), \Lambda_{r}(\lambda) x\left(\lambda^{d}\left\{t-\lambda^{-d} \tau\right\}, x_{0}\right)\right] \\
& =F\left[y\left(t, y_{0}\right), y\left(t-\lambda^{-d} \tau, y_{0}\right)\right] .
\end{aligned}
$$

Therefore, $y\left(t, y_{0}\right)$ is the system solution with initial conditions $y_{0}$ for another delay $\lambda^{-d} \tau$.

Corollary 1. Let the origin be locally asymptotically stable for a r-homogeneous system (1) with the degree $d=0$, then it is globally asymptotically stable.

Proof. Assume that the origin is locally attractive for (1) with an open domain of attraction $\mathcal{A} \subset C_{[-\tau, 0]}$, i.e. for any $\varepsilon>0$ and $x_{0} \in \mathcal{A}$ there is $T_{\varepsilon, x_{0}} \geq 0$ such that $\left\|x\left(t, x_{0}\right)\right\|_{r} \leq \varepsilon$ for all $t \geq T_{\varepsilon, x_{0}}$ (by Lemma 1 the norms $\|\cdot\|$ and $\|\cdot\|_{r}$ can be replaced). Take a $\mu>0$ such that $\mathcal{S}_{r}^{\mu} \subset \mathcal{A}$ where $\mathcal{S}_{r}^{\mu}=\mu \mathcal{S}_{r}$, then for any $\xi \in C_{[-\tau, 0]}$ there is $x_{0} \in \mathcal{S}_{r}^{\mu}$ such that $\xi=\Lambda_{r}(\lambda) x_{0}$ for $\lambda=\mu^{-1}\|\xi\|_{r}$ and the corresponding unique solution $x(t, \xi)=\Lambda_{r}(\lambda) x\left(\lambda^{d} t, x_{0}\right)$ by Proposition 1. Obviously, if $x\left(t, x_{0}\right) \rightarrow 0$ for all $x_{0} \in \mathcal{S}_{r}^{\mu}$ with $t \rightarrow+\infty$, then so is $x(t, \xi)=\Lambda_{r}(\lambda) x\left(\lambda^{d} t, x_{0}\right)$, and the claims about global attractiveness and forward completeness follow. 
To prove that local stability of the origin implies global one in this case, assume that $\sup _{t \geq 0}\left\|x\left(t, x_{0}\right)\right\|_{r} \leq \sigma\left(\left\|x_{0}\right\|_{r}\right)$ for all $x_{0} \in \mathcal{A}$ and some $\sigma \in \mathcal{K}$. Now take any $\xi \in C_{[-\tau, 0]}$, then there is $x_{0} \in \mathcal{S}_{r}^{\mu} \subset \mathcal{A}$ such that $\xi=\Lambda_{r}(\lambda) x_{0}$ for $\lambda=\mu^{-1}\|\xi\|_{r}$ with the corresponding unique solution $x(t, \xi)=\Lambda_{r}(\lambda) x\left(\lambda^{d} t, x_{0}\right)$ by Proposition 1. Therefore

$$
\begin{aligned}
\sup _{t \geq 0}\|x(t, \xi)\|_{r} & =\sup _{t \geq 0}\left\|\Lambda_{r}(\lambda) x\left(\lambda^{d} t, x_{0}\right)\right\|_{r}=\lambda \sup _{t \geq 0}\left\|x\left(\lambda^{d} t, x_{0}\right)\right\|_{r} \\
& \leq\|\xi\|_{r} \sigma\left(\left\|x_{0}\right\|_{r}\right) / \mu=\sigma(\mu) / \mu\|\xi\|_{r}
\end{aligned}
$$

and the system is Lyapunov stable $[19,23,29]$.

Thus we have proven that for homogeneous time-delay systems with $d=0$ any local stability/attractivity properties at the origin for a given delay $\tau$ hold globally. The case of homogeneous systems with $d=0$ becomes important for stability analysis using local homogeneous approximating dynamics, which are considered in Section 4 below (the approximating dynamics degree can be assigned to be zero). Apparently, for the case $d \neq 0$ even more interesting conclusions can be obtained for homogeneous systems.

Lemma 2. Let the system (1) be r-homogeneous with degree $d \neq 0$ and globally asymptotically stable for some delay $0<\tau_{0}<+\infty$, then it is globally asymptotically stable for any delay $0<\tau<+\infty$.

Proof. In this case for all $x_{0} \in C_{\left[-\tau_{0}, 0\right]}$ there is a function $\sigma \in \mathcal{K}$ such that $\left|x\left(t, x_{0}\right)\right|_{r} \leq \sigma\left(\left\|x_{0}\right\|_{r}\right)$ for all $t \geq 0$ and $\lim _{t \rightarrow+\infty}\left|x\left(t, x_{0}\right)\right|_{r}=0$. Take some $\tau \in(0,+\infty)$ and select an initial condition $y_{0} \in C_{[-\tau, 0]}$, then for $\lambda=$ $\left(\frac{\tau_{0}}{\tau}\right)^{1 / d}$ (this $\lambda$ is well defined since $d \neq 0$ ) there exists $x_{0} \in C_{\left[-\tau_{0}, 0\right]}$ such that $y_{0}(s)=\Lambda_{r}(\lambda) x_{0}\left(\lambda^{d} s\right)$ for $s \in[-\tau, 0]$, and $y\left(t, y_{0}\right)=\Lambda_{r}(\lambda) x\left(\lambda^{d} t, x_{0}\right)$ for all $t \geq 0$ by Proposition 1. Thus $\lim _{t \rightarrow+\infty}\left|y\left(t, y_{0}\right)\right|_{r}=\lambda \lim _{t \rightarrow+\infty}\left|x\left(\lambda^{d} t, x_{0}\right)\right|_{r}=$ $\lambda \lim _{t \rightarrow+\infty}\left|x\left(t, x_{0}\right)\right|_{r}=0$ and the solution $y\left(t, y_{0}\right)$ is converging asymptotically to the origin. In addition, $\left|y\left(t, y_{0}\right)\right|_{r}=\lambda\left|x\left(\lambda^{d} t, x_{0}\right)\right|_{r} \leq \lambda \sigma\left(\left\|x_{0}\right\|_{r}\right)=$ $\lambda \sigma\left(\lambda^{-1}|| y_{0} \|_{r}\right)$ for all $t \geq 0$, which implies stability of the system (1) for the delay $\tau$. The proven convergence to the origin and stability give the global asymptotic stability of the system for an arbitrary delay $\tau \in(0,+\infty)$.

It is a well known fact for linear systems (homogeneous systems of degree $d=$ 0 ) that its stability for a sufficiently small delay does not imply stability for all $\tau \in(0,+\infty)$ (the Corollary 1 has been proven for a fixed delay $\tau$ ). For nonlinear homogeneous systems with degree $d \neq 0$ this is not the case, according to the result of Lemma 2 if they are globally stable for some delay, then they can preserve their stability for an arbitrary delay $\tau \in(0,+\infty)$. This is a surprising advantage of this class of "nonlinear" time-delay systems.

Further let us consider several useful consequences of Proposition 1 and Lemma 2.

Corollary 2. Let the system (1) be r-homogeneous with degree d and asymptotically stable into the set $\Omega=B_{\rho}^{\tau}=\left\{\phi \in C_{[-\tau, 0]}:\|\phi\|_{r} \leq \rho\right\}$ for some $0<\rho<+\infty$ 
for any value of delay $0 \leq \tau<+\infty$, then it is globally asymptotically stable independently of delay.

Proof. For any $\tau>0$ take $y_{0} \in C_{[-\tau, 0]}, y_{0} \notin B_{\rho}^{\tau}$, then according to Proposition 1 there is $0<\lambda<+\infty\left(\lambda=\rho^{-1}\left\|y_{0}\right\|_{r}\right)$ and $x_{0} \in B_{\rho}^{\lambda^{d} \tau}$ such that $y_{0}(s)=$ $\Lambda_{r}(\lambda) x_{0}\left(\lambda^{d} s\right)$ for $s \in[-\tau, 0]$ and $y\left(t, y_{0}\right)=\Lambda_{r}(\lambda) x\left(\lambda^{d} t, x_{0}\right)$ for all $t \geq 0$. Since $x(t)$ converges asymptotically to the origin, the same property is satisfied for $y(t)$ and it enters the set $B_{\rho}^{\tau}$ in a finite time.

Thus for the case $d \neq 0$ the result of Corollary 1 can be obtained if local stability holds independently of delay, then (1) is globally asymptotically stable.

For ordinary differential equations it has been also shown that asymptotically stable/unstable homogeneous systems always have homogeneous Lyapunov functions $[2,13,35]$. In this work we would like to prove a similar (sufficient only) result for time-delay homogeneous systems. For time-delay systems there exist two main techniques for stability analysis based on the Lyapunov approach. The first one is based on Lyapunov-Krasovskii functionals, another one on Lyapunov-Razumikhin functions $[19,23,29]$. Unfortunately, due to peculiarities of the directional derivatives for functionals the Lyapunov-Krasovskii approach is hard to develop using homogeneity for a general case: the derivative of a homogeneous functional may be non-homogeneous $[14,15]$.

The Razumikhin approach is based on Lyapunov-Razumikhin functions defined on $\mathbb{R}^{n}[19,23,29]$, which give a pointwise sufficient criteria for stability (not a functional one). It allows us to develop this approach using homogeneous arguments.

Note, that if a function $V: \mathbb{R}^{n} \rightarrow \mathbb{R}_{+}$is positive definite and radially unbounded, then there are functions $\alpha_{1}, \alpha_{2} \in \mathcal{K}_{\infty}$ such that $\alpha_{1}(|x|) \leq V(x) \leq \alpha_{2}(|x|)$ for all $x \in \mathbb{R}^{n}$. If $V$ is $r$-homogeneous with a degree $\nu$, then for any $x \in \mathbb{R}^{n}$ we have $V(x)=V\left(\Lambda_{r}\left(|x|_{r}\right) y\right)=|x|_{r}^{\nu} V(y)$ for some $y \in \mathbb{S}_{r}$, therefore, in this case $\alpha_{1}(s)=$ $\left[\bar{\sigma}_{r}^{-1}(s)\right]^{\nu} \min _{y \in \mathbb{S}_{r}} V(y)$ and $\alpha_{2}(s)=\left[\underline{\sigma}_{r}^{-1}(s)\right]^{\nu} \max _{y \in \mathbb{S}_{r}} V(y)$, and $\nu$ should be strictly positive for radial unboundedness of $V$. In addition, if $V$ is continuously differentiable at the origin, then $\nu$ should be bigger than 1 .

Theorem 1. Let the function $f$ in (1) be $r$-homogeneous with degree $d \geq-\min _{1 \leq i \leq n} r_{i}$ and there exist a locally Lipschitz continuous r-homogeneous Lyapunov-Razumikhin function $V: \mathbb{R}^{n} \rightarrow \mathbb{R}_{+}$with degree $\nu>\max \{0,-d\}$ such that

(i) $\min _{y \in \mathbb{S}_{r}} V(y)>0$ and there exist functions $\alpha, \gamma \in \mathcal{K}$ such that for all $\varphi \in \mathcal{S}_{r}$

$$
\max _{\theta \in[-\tau, 0]} V[\varphi(\theta)]<\gamma\{V[\varphi(0)]\} \Rightarrow D^{+} V[\varphi(0)] f(\varphi) \leq-\alpha(|\varphi(0)|) ;
$$

(ii) there exists a function $\wp \in \mathcal{K}$ such that $\lambda s<\wp(\lambda s) \leq \lambda \gamma(s)$ for all $s, \lambda \in$ $\mathbb{R}_{+} \backslash\{0\}$.

Then the origin is globally asymptotically stable for the system (1).

Proof. The dilation transformation $\phi=\Lambda_{r}(\lambda) \varphi$ connects any $\phi \in C_{[-\tau, 0]} \backslash\{0\}$ with some $\varphi \in \mathcal{S}_{r}$ for properly chosen $\lambda>0$. Let us multiply the left-hand part of the implication (i) by $\lambda^{\nu}$ and the right-hand part by $\lambda^{\nu+d}$ : 
$\max _{\theta \in[-\tau, 0]} \lambda^{\nu} V[\varphi(\theta)]<\lambda^{\nu} \gamma\{V[\varphi(0)]\} \Rightarrow \lambda^{\nu+d} D^{+} V[\varphi(0)] f(\varphi) \leq-\lambda^{\nu+d} \alpha(|\varphi(0)|)$,

the inequalities and relations should not be changed for $\lambda>0$. Owing the functions $\gamma$ and $\wp$ properties, the inequality $\wp\left\{\lambda^{\nu} V[\varphi(0)]\right\} \leq \lambda^{\nu} \gamma\{V[\varphi(0)]\}$ holds. Formally $\inf _{\varphi \in \mathcal{S}_{r}}\{\alpha(|\varphi(0)|)\}=0$, however having in mind that $\varphi$ is a continuous function and a solution of (1), there exists

$$
a=\inf _{\varphi \in \mathcal{S}_{r}, \max _{\theta \in[-\tau, 0]} V[\varphi(\theta)]<\gamma\{V[\varphi(0)]\}} \alpha(|\varphi(0)|)
$$

and $a>0$. Indeed, for the case $\max _{\theta \in[-\tau, 0]} V[\varphi(\theta)]<\gamma\{V[\varphi(0)]\}$ we have

$$
\begin{aligned}
\alpha_{1}\left(\underline{\rho}_{r}(1)\right) & \leq \alpha_{1}(\| \varphi||)=\alpha_{1}\left(\max _{\theta \in[-\tau, 0]}|\varphi(\theta)|\right) \\
& =\max _{\theta \in[-\tau, 0]} \alpha_{1}(|\varphi(\theta)|) \leq \max _{\theta \in[-\tau, 0]} V(\varphi(\theta))<\gamma[V(\varphi(0))],
\end{aligned}
$$

thus $\alpha_{2}^{-1} \circ \gamma^{-1} \circ \alpha_{1}\left(\underline{\rho}_{r}(1)\right)<|\varphi(0)|$ for all such $\varphi \in \mathcal{S}_{r}$ and $a \geq \alpha_{2}^{-1} \circ \gamma^{-1} \circ$ $\alpha_{1}\left(\underline{\rho}_{r}(1)\right)>0$. Therefore, due to homogeneity of the functions $f$ and $V$ we have:

$$
\begin{gathered}
\max _{\theta \in[-\tau, 0]} V\left[\Lambda_{r}(\lambda) \varphi(\theta)\right]<\wp\left\{V\left[\Lambda_{r}(\lambda) \varphi(0)\right]\right\} \Rightarrow \\
D^{+} V\left[\Lambda_{r}(\lambda) \varphi(0)\right] f\left(\Lambda_{r}(\lambda) \varphi\right) \leq-\lambda^{\nu+d} a,
\end{gathered}
$$

or equivalently $\left(\lambda=|| \phi||_{r} \geq|\phi(0)|_{r} \geq \bar{\sigma}_{r}^{-1}(|\phi(0)|)\right)$,

$$
\max _{\theta \in[-\tau, 0]} V[\phi(\theta)]<\wp\{V[\phi(0)]\} \Rightarrow D^{+} V[\phi(0)] f(\phi) \leq-\alpha^{\prime}(|\phi(0)|),
$$

where the function $\alpha^{\prime}(s)=\left(\bar{\sigma}_{r}^{-1}(s)\right)^{\nu+d} a$ is from class $\mathcal{K}$ since $\nu+d>0$. Therefore, if Razumikhin arguments are true for $\varphi \in \mathcal{S}_{r}$, then they are valid for any $\phi \in C_{[-\tau, 0]}$, that implies the global asymptotic stability of the origin for (1) $[19,23,29]$.

The condition (i) imposed in Theorem 1 on the system (1) behavior is the conventional Razumikhin condition (except that in the homogeneous case it can be verified on the sphere $\mathcal{S}_{r}$ only). The constraint (ii) on existence of the function $\wp$ is new, for example, it is satisfied for any $\gamma(s)>s>0$.

Remark 1. Note that the full derivative $D^{+} V[\varphi(0)] f(\varphi)$ is a function of $\varphi \in \mathcal{S}_{r}$, however under the condition $\max _{\theta \in[-\tau, 0]} V\left[\Lambda_{r}(\lambda) \varphi(\theta)\right]<\wp\left\{V\left[\Lambda_{r}(\lambda) \varphi(0)\right]\right\}$ the inequality $D^{+} V[\varphi(0)] f(\varphi)<m(\varphi(0))$ holds for some $m: \mathbb{R}^{n} \rightarrow \mathbb{R}$. If the function $m$ is $r$-homogeneous (the functions $V$ and $f$ possess this property), then the property $m(\varphi(0)) \leq-\alpha(|\varphi(0)|)$ in (ii) has to be verified for $\varphi(0) \in \mathbb{S}_{r}$ only.

Instability conditions in the Lyapunov-Razumikhin framework have been formulated in $[21,22]$. We will say that the system (1) is unstable at the origin if for any $\delta>0$ there exist $\varepsilon>0,\left\|x_{0}\right\| \leq \delta$ and $t_{x_{0}, \varepsilon}^{\prime} \geq 0$ such that $\left\|x_{t_{x_{0}, \varepsilon}^{\prime}}\right\|>\varepsilon$. For a function $V: \mathbb{R}^{n} \rightarrow \mathbb{R}_{+}$define two sets: 


$$
\begin{aligned}
& P_{M}^{V}=\left\{\varphi \in \mathcal{S}_{r}: V[\varphi(0)]=\max _{\theta \in[-\tau, 0]} V[\varphi(\theta)]\right\}, \\
& P_{m}^{V}=\left\{\varphi \in \mathcal{S}_{r}: V[\varphi(0)]=\min _{\theta \in[-\tau, 0]} V[\varphi(\theta)]\right\} .
\end{aligned}
$$

Theorem 2. Let the function $f$ in (1) be $r$-homogeneous with degree $d \geq-\min _{1 \leq i \leq n} r_{i}$ and there exist a locally Lipschitz continuous r-homogeneous Lyapunov-Razumikhin function $V: \mathbb{R}^{n} \rightarrow \mathbb{R}_{+}$with degree $\nu>\max \{0,-d\}$ such that $\min _{y \in \mathbb{S}_{r}} V(y)>0$ and one of the following properties is satisfied:

(i) $D^{-} V[\varphi(0)] f(\varphi)>0$ for all $\varphi \in P_{M}^{V}$;

(ii) $D^{-} V[\varphi(0)] f(\varphi)>0$ for all $\varphi \in P_{m}^{V}$.

Then the system (1) is unstable at the origin.

Proof. Select a $\lambda>0$ such that the dilation transformation $\phi=\Lambda_{r}(\lambda) \varphi$ connects a $\phi \in C_{[-\tau, 0]} \backslash\{0\}$ with some $\varphi \in \mathcal{S}_{r}$. The sets $\Pi_{M}^{V}=\left\{\phi \in C_{[-\tau, 0]}: V[\phi(0)]=\right.$ $\left.\max _{\theta \in[-\tau, 0]} V[\phi(\theta)]\right\}, \Pi_{m}^{V}=\left\{\phi \in C_{[-\tau, 0]}: V[\phi(0)]=\min _{\theta \in[-\tau, 0]} V[\phi(\theta)]\right\}$ are well defined by the dilation transformation of $P_{M}^{V}, P_{m}^{V}$ :

$$
\begin{aligned}
\cup_{\lambda>0} \Lambda_{r}(\lambda) P_{M}^{V} & =\left\{\phi=\Lambda_{r}(\lambda) \varphi, \lambda>0, \varphi \in \mathcal{S}_{r}: V[\varphi(0)]=\max _{\theta \in[-\tau, 0]} V[\varphi(\theta)]\right\} \\
& =\left\{\phi=\Lambda_{r}(\lambda) \varphi, \lambda>0, \varphi \in \mathcal{S}_{r}: \lambda^{\nu} V[\varphi(0)]=\lambda^{\nu} \max _{\theta \in[-\tau, 0]} V[\varphi(\theta)]\right\} \\
& =\Pi_{M}^{V}, \\
\cup_{\lambda>0} \Lambda_{r}(\lambda) P_{m}^{V} & =\left\{\phi=\Lambda_{r}(\lambda) \varphi, \lambda>0, \varphi \in \mathcal{S}_{r}: V[\varphi(0)]=\min _{\theta \in[-\tau, 0]} V[\varphi(\theta)]\right\} \\
& =\left\{\phi=\Lambda_{r}(\lambda) \varphi, \lambda>0, \varphi \in \mathcal{S}_{r}: \lambda^{\nu} V[\varphi(0)]=\lambda^{\nu} \min _{\theta \in[-\tau, 0]} V[\varphi(\theta)]\right\} \\
& =\Pi_{m}^{V} .
\end{aligned}
$$

Take $\phi \in \Pi_{M}^{V}$ or $\phi \in \Pi_{m}^{V}$, then

$$
D^{-} V[\phi(0)] f(\phi)=\lambda^{\nu+d} D^{-} V[\varphi(0)] f(\varphi)>0 .
$$

Therefore, $D^{-} V[\phi(0)] f(\phi)>0$ for all $\phi \in \Pi_{M}^{V}$ or $\phi \in \Pi_{m}^{V}$, which are the conditions of instability of (1) into the sets $\Pi_{M}^{V}$ or $\Pi_{m}^{V}$ from [21,22].

The results of theorems 1 and 2 mean that by using homogeneous LyapunovRazumikhin functions the global stability/instability of a homogeneous system at the origin can be checked on the sphere $\mathcal{S}_{r}$ only. These facts may simplify the function $V$ search and the system analysis with application of a numerical routine. The drawback is that in the space $C_{[-\tau, 0]}$, the sphere $\mathcal{S}_{r}$ is a rather complex object.

\section{Local homogeneity}

A disadvantage of the global homogeneity introduced so far is that such systems possess the same behavior "globally". Thus the homogeneous systems are not re- 
ally "nonlinear", they have similar diversity of operating modes as linear systems. In fact, from analysis and design points of view the homogeneous systems are a generalization of linear ones. Comparing with other nonlinear systems, it may be easier to find a (homogeneous) Lyapunov function for homogeneous systems. That is why finding a possibility to apply this approach for a broader class of nonlinear systems is very important.

An approach to resolve this issue consists in introducing a local version of homogeneity as in [13].

Definition 2. The function $g: C_{[-\tau, 0]} \rightarrow \mathbb{R}$ is called $\left(r, \lambda_{0}, g_{0}\right)$-homogeneous $\left(r_{i}>\right.$ $\left.0, i=\overline{1, n} ; g_{0}: C_{[-\tau, 0]} \rightarrow \mathbb{R}\right)$ if for any $\phi \in \mathcal{S}_{r}$ the relation

$$
\lim _{\lambda \rightarrow \lambda_{0}} \lambda^{-d_{0}} g\left(\Lambda_{r}(\lambda) \phi\right)-g_{0}(\phi)=0
$$

is satisfied (uniformly on $\mathcal{S}_{r}$ for $\lambda_{0} \in\{0,+\infty\}$ ) for some $d_{0} \in \mathbb{R}$.

The system (1) is called $\left(r, \lambda_{0}, f_{0}\right)$-homogeneous $\left(r_{i}>0, i=\overline{1, n} ; f_{0}\right.$ : $\left.C_{[-\tau, 0]} \rightarrow \mathbb{R}^{n}\right)$ if for any $\phi \in \mathcal{S}_{r}$ the relation

$$
\lim _{\lambda \rightarrow \lambda_{0}} \lambda^{-d_{0}} \Lambda_{r}^{-1}(\lambda) f\left(\Lambda_{r}(\lambda) \phi\right)-f_{0}(\phi)=0
$$

is satisfied (uniformly on $\mathcal{S}_{r}$ for $\lambda_{0} \in\{0,+\infty\}$ ) for some $d_{0} \geq-\min _{1 \leq i \leq n} r_{i}$.

For a given $\lambda_{0}, g_{0}$ and $f_{0}$ are called approximating functions.

For any $0<\lambda_{0}<+\infty$ the following formulas give a variant of homogeneous approximating functions $g_{0}$ and $f_{0}$ :

$$
\begin{aligned}
& g_{0}(\phi)=\|\phi\|_{r}^{d} \lambda_{0}^{-d_{0}} g\left(\Lambda_{r}\left(\lambda_{0}\right) \Lambda_{r}^{-1}\left(\|\phi\|_{r}\right) \phi\right), d \geq 0, \\
& f_{0}(\phi)=\|\phi\|_{r}^{d} \lambda_{0}^{-d_{0}} \Lambda_{r}\left(\|\phi\|_{r}\right) \Lambda_{r}^{-1}\left(\lambda_{0}\right) f\left(\Lambda_{r}\left(\lambda_{0}\right) \Lambda_{r}^{-1}\left(\|\phi\|_{r}\right) \phi\right), d \geq-\min _{1 \leq i \leq n} r_{i} .
\end{aligned}
$$

This property is called local homogeneity [13], it allows us to analyze local stability/instability of the system (1) on the basis of a simplified system

$$
d y(t) / d t=f_{0}\left[y_{\tau}(t)\right], t \geq 0,
$$

called the local approximating dynamics for (1).

Theorem 3. [15] Let the system (1) be ( $\left.r, \lambda_{0}, f_{0}\right)$-homogeneous for some $r_{i}>0, i=$ $\overline{1, n}$, the function $f_{0}$ be continuous and $r$-homogeneous with the degree $d_{0}$. Suppose there exists a locally Lipschitz continuous r-homogeneous Lyapunov-Razumikhin function $V_{0}: \mathbb{R}^{n} \rightarrow \mathbb{R}_{+}$with the degree $\nu_{0}>\max \left\{0,-d_{0}\right\}, \alpha_{1}(|x|) \leq V_{0}(x) \leq$ $\alpha_{2}(|x|)$ for all $x \in \mathbb{R}^{n}$ and some $\alpha_{1}, \alpha_{2} \in \mathcal{K}_{\infty}$ such that:

(i) there exist functions $\alpha, \gamma \in \mathcal{K}$ such that for all $\varphi \in \mathcal{S}_{r}$

$$
\max _{\theta \in[-\tau, 0]} V_{0}[\varphi(\theta)]<\gamma\left\{V_{0}[\varphi(0)]\right\} \Rightarrow D^{+} V_{0}[\varphi(0)] f_{0}(\varphi) \leq-\alpha(|\varphi(0)|) ;
$$


(ii) there exists a function $\wp \in \mathcal{K}$ such that $\lambda s<\wp(\lambda s) \leq \lambda \gamma(s)$ for all $s, \lambda \in$ $\mathbb{R}_{+} \backslash\{0\}$.

Then

1) if $\lambda_{0}=0$, then there exists $0<\bar{\lambda}_{\varepsilon}$ such that the system (1) is locally asymptotically stable at the origin with the domain of attraction containing the set

$$
X_{0}=\left\{\phi \in C_{[-\tau, 0]}:\|\phi\| \leq \alpha_{1}^{-1} \circ \alpha_{2} \circ \bar{\rho}_{r}\left(\bar{\lambda}_{\varepsilon}\right)\right\}
$$

2) if $\lambda_{0}=+\infty$, then there exists $0<\underline{\lambda}_{\varepsilon}<+\infty$ such that the system (1) is globally asymptotically stable with respect to forward invariant set

$$
X_{\infty}=\left\{\phi \in C_{[-\tau, 0]}:\|\phi\| \leq \alpha_{1}^{-1} \circ \alpha_{2} \circ \underline{\rho}_{r}\left(\underline{\lambda}_{\varepsilon}\right)\right\} ;
$$

3) if $0<\lambda_{0}<+\infty$, then there exist $0<\underline{\lambda}_{\varepsilon} \leq \lambda_{0} \leq \bar{\lambda}_{\varepsilon}<+\infty$ such that the system (1) is asymptotically stable with respect to the forward invariant set $X_{\infty}$ with region of attraction

$$
\begin{aligned}
X= & \left\{\phi \in C_{[-\tau, 0]}: \alpha_{1}^{-1} \circ \alpha_{2} \circ \underline{\rho}_{r}\left(\underline{\lambda}_{\varepsilon}\right)<\|\phi\|\right. \\
& \left.<\alpha_{1}^{-1} \circ \alpha_{2} \circ \bar{\rho}_{r}\left(\bar{\lambda}_{\varepsilon}\right)\right\}
\end{aligned}
$$

provided that the set $X$ is connected and nonempty.

Theorem 4. [15] Let the system (1) be ( $\left.r, \lambda_{0}, f_{0}\right)$-homogeneous for some $r_{i}>0, i=$ $\overline{1, n}$, the function $f_{0}$ be continuous and $r$-homogeneous with the degree $d_{0}$. Suppose there exists a locally Lipschitz continuous r-homogeneous Lyapunov-Razumikhin function $V_{0}: \mathbb{R}^{n} \rightarrow \mathbb{R}_{+}$with the degree $\nu_{0}>\max \left\{0,-d_{0}\right\}, \alpha_{1}(|x|) \leq V_{0}(x) \leq$ $\alpha_{2}(|x|)$ for all $x \in \mathbb{R}^{n}$ and some $\alpha_{1}, \alpha_{2} \in \mathcal{K}_{\infty}$, such that $D^{-} V_{0}[\varphi(0)] f_{0}(\varphi) \geq a>$ 0 for all $\varphi \in P_{m}^{V_{0}}$ or $\varphi \in P_{M}^{V_{0}}$. Then

1) if $\lambda_{0}=0$, then there exists $0<\bar{\lambda}_{\varepsilon}$ such that for the system (1) the set

$$
X_{0}=\left\{\phi \in C_{[-\tau, 0]}:\|\phi\| \leq \alpha_{1}^{-1} \circ \alpha_{2} \circ \bar{\rho}_{r}\left(\bar{\lambda}_{\varepsilon}\right)\right\}
$$

is unstable;

2) if $\lambda_{0}=+\infty$, then there exists $0<\underline{\lambda}_{\varepsilon}<+\infty$ such that for the system (1) the set

$$
X_{\infty}=\left\{\phi \in C_{[-\tau, 0]}:\|\phi\| \leq \alpha_{1}^{-1} \circ \alpha_{2} \circ \underline{\rho}_{r}\left(\underline{\lambda}_{\varepsilon}\right)\right\}
$$

is unstable;

3) if $0<\lambda_{0}<+\infty$, then there exist $0<\underline{\lambda}_{\varepsilon} \leq \lambda_{0} \leq \bar{\lambda}_{\varepsilon}<+\infty$ such that for the system (1) the set $X_{\infty}$ is unstable provided that the set $X=\left\{\phi \in C_{[-\tau, 0]}\right.$ : $\left.\alpha_{1}^{-1} \circ \alpha_{2} \circ \underline{\rho}_{r}\left(\underline{\lambda}_{\varepsilon}\right)<\|\phi\|<\alpha_{1}^{-1} \circ \alpha_{2} \circ \bar{\rho}_{r}\left(\bar{\lambda}_{\varepsilon}\right)\right\}$ is connected and non empty.

These results establish the links between different variants of local homogeneity with stable/unstable approximating dynamics (3) and the stability/instability properties of the original system (1), similarly to [13].

Corollary 3. [15] Let the system (1) be $\left(r, \lambda_{0}, f_{0}\right)$-homogeneous for some $r_{i}>0$, $i=\overline{1, n}$, the function $f_{0}: R^{n} \rightarrow \mathbb{R}^{n}$ be continuous and $r$-homogeneous with 
the degree $d_{0}$ and there exist an $r$-homogeneous Lyapunov-Razumikhin function $V_{0}: \mathbb{R}^{n} \rightarrow \mathbb{R}_{+}$with the degree $\nu_{0}>\max \left\{0,-d_{0}\right\}$ such that $D^{+} V_{0}(x) f_{0}(x) \leq$ $-\alpha(|x|)\left(D^{-} V_{0}(x) f_{0}(x) \geq \alpha(|x|)\right)$ for all $x \in \mathbb{S}_{r}$ with $\alpha \in \mathcal{K}$. Then all conclusions of Theorem 3 (Theorem 4) hold.

Similarly to [13], one can use these conditions to detect for (1) the presence of Yakubovich oscillations [12].

\section{ISS property of time-delay homogeneous systems}

Consider the system (1) with inputs:

$$
d x(t) / d t=f\left[x_{t}, u(t)\right], t \geq 0,
$$

where $x \in \mathbb{R}^{n}, x_{t} \in C_{[-\tau, 0]}$ is the state function as before, and $u: \mathbb{R}_{+} \rightarrow \mathbb{R}^{m}$ is an essentially bounded (Lebesgue) measurable input, $\|u\|_{\infty}=e s s \cdot \sup _{t \geq 0}|u(t)|$ (we will denote by $\mathcal{L}_{\infty}$ the set of inputs $u: \mathbb{R}_{+} \rightarrow \mathbb{R}^{m}$ with $\|u\|_{\infty}<+\infty$ ); $f: C_{[-\tau, 0]} \times \mathbb{R}^{m} \rightarrow \mathbb{R}^{n}$ is a continuous function (locally Lipschitz with respect to $\left.x_{t}\right), f(0,0)=0$. Under these conditions the system (4) has a unique solution $x\left(t, x_{0}, u\right)$ for any $u \in \mathcal{L}_{\infty}$ and $x_{0} \in C_{[-\tau, 0]}$ defined on some interval $[-\tau, T)$.

The Lyapunov-Razumikhin theory has been extended to the ISS notion analysis in [39] as follows.

Definition 3. The system (4) is called ISS, if for all $x_{0} \in C_{[-\tau, 0]}$ and $u \in \mathcal{L}_{\infty}$ there exist $\beta \in \mathcal{K} \mathcal{L}$ and $\gamma \in \mathcal{K}$ such that for all $t \geq 0$ :

$$
\left|x\left(t, x_{0}, u\right)\right| \leq \beta\left(\left\|x_{0}\right\|, t\right)+\gamma\left(\|u\|_{\infty}\right) .
$$

Theorem 5. Let for the system (4) there exist a locally Lipschitz continuous ISS Lyapunov-Razumikhin function $V: \mathbb{R}^{n} \rightarrow \mathbb{R}_{+}$, i.e. there are functions $\alpha_{1}, \alpha_{2}, \alpha_{3} \in$ $\mathcal{K}_{\infty}$ and $\gamma, \chi \in \mathcal{K}\left(\gamma(s)>s\right.$ for all $\left.s \in \mathbb{R}_{+}\right)$such that for all $x \in \mathbb{R}^{n}, \varphi \in C_{[-\tau, 0]}$ and $u \in \mathbb{R}^{m}$

$$
\begin{gathered}
\alpha_{1}(|x|) \leq V(x) \leq \alpha_{2}(|x|), \\
\max \left(\max _{\theta \in[-\tau, 0]} V[\varphi(\theta)], \chi(|u|)\right)<\gamma\{V[\varphi(0)]\} \Rightarrow D^{+} V[\varphi(0)] f(\varphi, u) \leq-\alpha_{3}(|\varphi(0)|) .
\end{gathered}
$$

Then the system (4) is ISS.

In $[6,26,37]$ it has been shown that if a nonlinear dynamical system is homogeneous, then it is also ISS with respect to an input (e.g. additive disturbance or measurement noise). A similar link for time-delay system (4) is established in the theorem below. Define $\tilde{f}\left(x_{t}, u\right)=\left[f\left(x_{t}, u\right)^{T} 0_{m}\right]^{T} \in \mathbb{R}^{n+m}$, it is an extended auxiliary vector field for the system (4), where $0_{m}$ is the zero vector with $m$ elements. 
Theorem 6. [15] Let the vector field $\tilde{f}$ be homogeneous with the weights $r=$ $\left[r_{1}, \ldots, r_{n}\right]>0, \tilde{r}=\left[\tilde{r}_{1}, \ldots, \tilde{r}_{m}\right]>0$ with a degree $d \geq-r_{\min }, r_{\min }=$ $\min _{1 \leq i \leq n} r_{i}$, i.e.

$$
f\left(\Lambda_{r}(\lambda) x_{t}, \Lambda_{\tilde{r}}(\lambda) u\right)=\lambda^{d} \Lambda_{r}(\lambda) f\left(x_{t}, u\right) \quad \forall \lambda>0 .
$$

Assume that for the system (4) for $u=0$ there exists a continuously differentiable $r$-homogeneous Lyapunov-Razumikhin function $V: \mathbb{R}^{n} \rightarrow \mathbb{R}_{+}, V(0)=0$ with degree $\nu>r_{\max }=\max _{1 \leq i \leq n} r_{i}$ such that:

(i) $\min _{y \in \mathbb{S}_{r}} V(y)>0$ and there exist functions $\alpha, \gamma \in \mathcal{K}$ such that for all $\varphi \in \mathcal{S}_{r}$

$$
\max _{\theta \in[-\tau, 0]} V[\varphi(\theta)]<\gamma\{V[\varphi(0)]\} \Rightarrow D^{+} V[\varphi(0)] f(\varphi, 0) \leq-\alpha(|\varphi(0)|) ;
$$

(ii) there exists function $\wp \in \mathcal{K}$ such that $\lambda s<\wp(\lambda s) \leq \lambda \gamma(s)$ for all $s, \lambda \in$ $\mathbb{R}_{+} \backslash\{0\}$.

Then the system (4) is ISS.

The result of Theorem 6 says that if the conditions of Theorem 1 are satisfied for the case $u=0$ for the system (4), then it is ISS. This conclusion highlights an additional importance of the introduced homogeneity concept for time-delay systems: under additional algebraic restrictions on the system equations and its LyapunovRazumikhin function (homogeneity) we gain the system robustness.

\section{Examples}

In this section we will consider several examples of homogeneous systems to illustrate various aspects of Proposition 1, theorems 3, 4 and 6.

\subsection{A homogeneous time-delay system with degree $d=0$}

Consider the system

$$
\dot{x}(t)=-2|x(t)|^{\alpha} \operatorname{sign}[x(t)]+|x(t-\tau)|^{\alpha} \operatorname{sign}[x(t-\tau)],
$$

where $x(t) \in \mathbb{R}, \tau>0$ is a fixed time delay, $\alpha>0$ is a parameter. Applying the Lyapunov-Razumikhin approach with $V(x)=0.5 x^{2}$ it is straightforward to show that the system is stable for any $\tau>0$. The system is homogeneous for $r=1$ and $d=\alpha-1$. According to Proposition 1 , if $x\left(t, x_{0}\right)$ is a solution of the system (1) with initial condition $x_{0} \in C_{[-\tau, 0]}$, then $y\left(t, y_{0}\right)=\Lambda_{r}(\lambda) x\left(\lambda^{d} t, x_{0}\right)$ is a solution of the system (2) with the initial condition $y_{0} \in C_{\left[-\lambda^{-d} \tau, 0\right]}, y_{0}(s)=\Lambda_{r}(\lambda) x_{0}\left(\lambda^{d} s\right)$ for $s \in\left[-\lambda^{-d} \tau, 0\right]$ for any $\lambda>0$. For $\tau=10$ and two values of $\alpha(\alpha=0.5$ and $\alpha=1.5)$, the system (1) trajectory $x\left(t, x_{0}\right)$ with $x_{0}(s)=2, s \in[-\tau, 0]$, the system 
(2) trajectory $z\left(t, z_{0}\right)$ for $z_{0}(s)=2, s \in\left[-3^{1-\alpha} \tau, 0\right]$ and $y(t)=3 x\left(3^{\alpha-1} t, x_{0}\right)$ are shown in Fig. 1. The results of these simulations confirm the scaling property established in Proposition 1 since the variables $y(t)$ and $z(t)$ coincide.

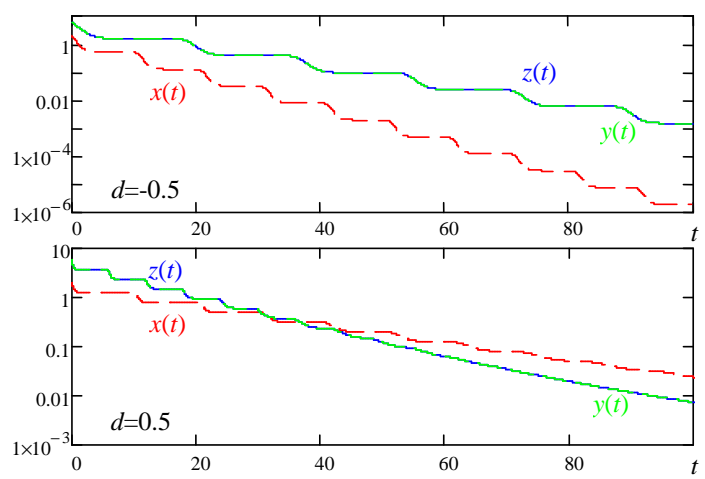

Fig. 1 Scaling of trajectories of a homogeneous system with different degrees

\subsection{Nicholson's blowfly population dynamics}

Consider a recruitment-delayed model of a population [20,33]:

$$
\dot{x}(t)=b x(t-\tau) e^{-c x(t-\tau)}-a x(t),
$$

where $x(t) \in \mathbb{R}_{+}$is the adult population size; $a, b$ and $c$ are some positive parameters; $\tau>0$ is a fixed time delay. The first term in the right-hand side represents the birth rate, and the second term corresponds to the death rate in the population. This equation was introduced by Nicholson [33] to model a laboratory fly population. The appearance of delay $\tau$ models the maturation period in the population after birth. This system is locally homogeneous in the bi-limit:

$$
\begin{gathered}
\lambda_{1}=0, r_{1}=1, f_{1}\left(x_{\tau}\right)=b x(t-\tau)-a x(t), d_{1}=0 ; \\
\lambda_{2}=+\infty, r_{2}=1, f_{2}\left(x_{\tau}\right)=-a x(t), d_{2}=0 .
\end{gathered}
$$

Usually it is assumed that $b>a$, then the approximation at the origin is unstable and an equilibrium different from zero exists: $\bar{x}=c^{-1} \ln \frac{b}{a}$. First approximation arguments show that for $b>a e^{2}$ the steady state $\bar{x}$ is also unstable for all $\tau \geq \tau_{0}$ for a sufficiently big critical delay $\tau_{0}>0$. Thus the system (3) at $\lambda_{1}=0$ is unstable, that according to Theorem 4 implies instability of the system around the origin. Obviously for $\lambda_{2}=+\infty$ the system (3) is asymptotically stable that, from Theorem 3 , ensures global convergence of the trajectories to a vicinity of the origin. Note 
that approximate behavior of the system in this case is defined by the delay-free dynamics (Corollary 3). Since a non trivial equilibrium $\bar{x}$ is also unstable, then this population dynamics is oscillating in the sense of Yakubovich [12]. The results of simulation for $b=15, a=1, \tau=10$ and $c=0.5$ are presented in Fig. 2 (two trajectories $x(t)$ are shown for different initial conditions).

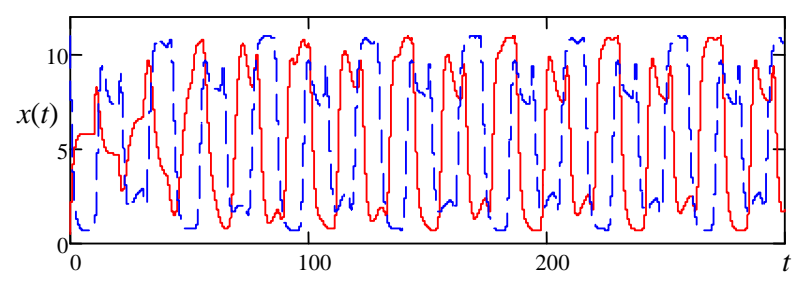

Fig. 2 The results of simulation for the Nicholson's population model

\subsection{An ISS academic example with degree $d \neq 0$}

Consider the following system (a modification of quasi-polynomial model widely used in the systems biology $[10,31])$ :

$$
\begin{aligned}
& \dot{x}_{1}(t)=-a_{1} x_{1}(t)\left[x_{1}(t)+u_{1}(t)\right]+b_{1} x_{2}(t-\tau)+u_{2}(t), \\
& \dot{x}_{2}(t)=-a_{2} x_{2}^{1.5}(t)+\left[b_{2} x_{2}(t)+u_{3}(t)\right] x_{1}(t-\tau),
\end{aligned}
$$

where $x_{1}(t) \in \mathbb{R}_{+}$and $x_{2}(t) \in \mathbb{R}_{+}$represent some concentrations in a genetic network, the inputs $u_{i}(t) \in \mathbb{R}_{+}, i=\overline{1,3}$ are the model uncertainties, $\tau>0$ represents the transition delay in the network; $a_{1}, a_{2}, b_{1}$ and $b_{2}$ are positive parameters. It is straightforward to check that the system is positive and homogeneous for $r=\left[\begin{array}{ll}1 & 2\end{array}\right]$ and $\tilde{r}=\left[\begin{array}{lll}1 & 2 & 2\end{array}\right]$ with degree $d=1$. For $u_{i}=0, i=\overline{1,3}$ consider a Lyapunov function $V\left(x_{1}, x_{2}\right)=x_{1}^{2}+x_{2}$, which is $r$-homogeneous and (note that $\max \left\{x_{1}^{2}, x_{2}\right\} \leq V\left(x_{1}, x_{2}\right)$ and $\left.V^{1.5}\left(x_{1}, x_{2}\right) \leq \frac{3}{2}\left[x_{1}^{3}+x_{2}^{1.5}\right]\right)$

$$
\begin{aligned}
\dot{V}(t)= & -2 a_{1} x_{1}^{3}(t)+2 b_{1} x_{1}(t) x_{2}(t-\tau)-a_{2} x_{2}^{1.5}(t)+b_{2} x_{1}(t-\tau) x_{2}(t) \\
\leq & -\min \left\{2 a_{1}, a_{2}\right\}\left[x_{1}^{3}(t)+x_{2}^{1.5}(t)\right] \\
& +\max \left\{2 b_{1}, b_{2}\right\}\left[x_{1}(t) x_{2}(t-\tau)+x_{1}(t-\tau) x_{2}(t)\right] \\
\leq & -\frac{2}{3} \min \left\{2 a_{1}, a_{2}\right\} V^{1.5}(t) \\
& +\max \left\{2 b_{1}, b_{2}\right\}[\sqrt{V(t)} V(t-\tau)+\sqrt{V(t-\tau)} V(t)] .
\end{aligned}
$$

Let

$$
\max \left\{2 b_{1}, b_{2}\right\}[p+\sqrt{p}]+\epsilon \leq \frac{2}{3} \min \left\{2 a_{1}, a_{2}\right\}
$$


for some $p>1$ and $\epsilon>0$, then

$$
p V(t) \geq V(t-\tau) \Rightarrow \dot{V}(t) \leq-\epsilon V^{1.5}(t)
$$

and $V$ is a Lyapunov-Razumikhin function of the unperturbed system. Then, by Theorem 6 , the system is ISS with respect to inputs $u_{i}(t), i=\overline{1,3}$. This conclusion is confirmed by the results of numerical simulation presented in Fig. 3 for

$$
\begin{gathered}
a_{1}=1, a_{2}=2, b_{1}=0.25, b_{2}=0.5, p=0.9 \\
u_{1}(t)=\sin ^{2}(0.5 t), u_{2}(t)=2 \cos ^{2}(2 t), u_{3}(t)=\sin ^{2}(t)
\end{gathered}
$$

and different values of $\tau$.
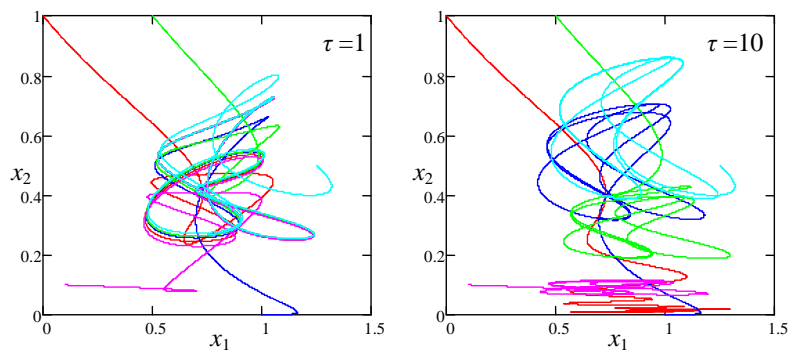

Fig. 3 Trajectories of an ISS system

\section{Conclusions}

A way how to develop the homogeneity notion to time-delay systems is presented. It is shown that a local stability notion for homogeneous time-delay systems holds globally, that simplifies analysis of such a type of systems applying linearization at the origin, for instance. The sufficient conditions for stability/instability of homogeneous systems are presented. These conditions are based on the Razumikhin stability arguments. It is also shown that if a homogeneous system has a homogeneous Lyapunov-Razumikhin function, then under a mild structural condition (dealing with degree of homogeneity) it is ISS. The definition of local homogeneity is proposed, relations between stability/instability of the locally approximating dynamics and the original system are established. Efficiency of the proposed approach is demonstrated on numerical experiments. 


\section{References}

1. Aleksandrov, A., and Zhabko, A.: On the asymptotic stability of solutions of nonlinear systems with delay. Siberian Mathematical Journal 53(3), 393-403 (2012)

2. Andrieu, V., Praly, L., and Astolfi, A.: Homogeneous approximation, recursive observer design, and output feedback. SIAM J. Control Optimization 47(4), 1814-1850 (2008)

3. Asl, F., and Ulsoy, A.: Analytical solution of a system of homogeneous delay differential equations via the Lambert function. In: Proc. American Control Conference (Chicago), pp. 24962500 (2000)

4. Bacciotti, A., and Rosier, L.: Liapunov Functions and Stability in Control Theory. Lecture Notes in Control and Inform. Sci. Springer, Berlin (2001)

5. Bernuau, E., Polyakov, A., Efimov, D., and Perruquetti, W.: Verification of ISS, iISS and IOSS properties applying weighted homogeneity. Systems \& Control Letters 62(12), 1159-1167 (2013)

6. Bernuau, E., Polyakov, A., Efimov, D., and Perruquetti, W.: On ISS and iISS properties of homogeneous systems. In: Proc. European Control Conference (ECC) 2013 (Zürich) (2013)

7. Bhat, S., and Bernstein, D.: Geometric homogeneity with applications to finite-time stability. Mathematics of Control, Signals and Systems 17, 101-127 (2005)

8. Bokharaie, V., Mason, O., and Verwoerd, M.: D-stability and delay-independent stability of homogeneous cooperative systems. IEEE Trans. Automatic Control 55(12), 2882-2885 (2010)

9. Chiasson, J., and Loiseau, J. (eds.): Applications of Time Delay Systems. Lecture Notes in Control and Information Sciences. Springer (2007)

10. Chowdhury, A. R., Chetty, M., and Vinh, N. X.: Incorporating time-delays in S-System model for reverse engineering genetic networks. BMC Bioinformatics 14, 196 (2013)

11. Dublik, J.: Asymptotic equilibrium for homogeneous delay linear differential equations with 1-perturbation term. Nonlinear Analysis, Theory, Methods \& Applicarions 30(6), 3927-3933 (1997)

12. Efimov, D., and Fradkov, A.: Oscillatority conditions for nonlinear systems with delays. Journal of Applied Mathematics, Article ID 72561, 1-12 (2007)

13. Efimov, D., and Perruquetti, W.: Oscillations conditions in homogeneous systems. In: Proc. NOLCOS'10 (Bologna), pp. 1379-1384 (2010)

14. Efimov, D., and Perruquetti, W.: Homogeneity for time-delay systems. In: Proc. IFAC WC 2011, (Milan) (2011)

15. Efimov, D., Perruquetti, W., and Richard, J.-P.: Development of homogeneity concept for timedelay systems. SIAM J. Control Optim. 52(3), 1403-1808 (2014)

16. Erneux, T.: Applied Delay Differential Equations. Springer, NY (2009)

17. Fridman, E.: A refined input delay approach to sampled-data control. Automatica 46, 421-427 (2010)

18. Grüne, L.: Homogeneous state feedback stabilization of homogeneous systems. SIAM J. Control Optimization 38(4), 1288-1314 (2000)

19. Gu, K., Kharitonov, K., and Chen, J.: Stability of Time-Delay Systems. Control Engineering, Birkhäuser, Boston (2003)

20. Gurney, W. S. C., Blythe, S. P., and Nisbet, R. M.: Nicholsons blowfly revisited. Nature 287, 17-21 (1980)

21. Haddock, J., and Ko, Y.: Lyapunov-Razumikhin functions and an instability theorem for autonomous functional differential equations with finite delay. Rocky Mtn. J. Math. 25, 261-267 (1995)

22. Haddock, J., and Zhao, J.: Instability for autonomous and periodic functional differential equations with finite delay. Funkcialaj Ekvacioj 39, 553-570 (1996)

23. Hale, J.: Theory of Functional Differential Equations. Springer-Verlag (1977)

24. Hermes, H.: Nilpotent and high-order approximations of vector field systems. SIAM Review 33(2), 238-264 (1991)

25. Hermes, H.: Differential Equations: Stability and Control. Lecture Notes in Pure Appl. Math., ch. Homogeneous coordinates and continuous asymptotically stabilizing feedback controls, 249-260. Marcel Dekker (1991) 
26. Hong, $\mathrm{Y} .: \mathrm{H}_{\infty}$ control, stabilization, and input-output stability of nonlinear systems with homogeneous properties. Automatica 37(7), 819-829 (2001)

27. Hong, Y.: Finite-time stabilization and stabilizability of a class of controllable systems. Systems\& Control Lett. 46, 231-236 (2002)

28. Kawski, M.: Homogeneous feedback stabilization. Progress in systems and control theory: New trends in systems theory. Birkhäuser (1991)

29. Kolmanovsky, V., and Nosov, V.: Stability of functional differential equations. CA:Academic, San Diego (1986)

30. Ménard, T., Moulay, E., and Perruquetti, W.: Homogeneous approximations and local observer design. ESAIM: Control, Optimization and Calculus of Variations 19(3), 906-929 (2013)

31. Motee, N., Bamieh, B., and Khammash, M.: Stability analysis of quasi-polynomial dynamical systems with applications to biological network models. Automatica 48(6), 2945-2950 (2012)

32. Moulay, E., and Perruquetti, W.: Finite time stability and stabilization of a class of continuous systems. J. Mathematical Analysis Applications 323(2), 1430-1443 (2006)

33. Nicholson, A.: An outline of the dynamics of animal populations. Aust. J. Zool. 2, 9-65 (1954)

34. Richard, J.-P.: Time-delay systems: an overview of some recent advances and open problems. Automatica 39, 1667-1694 (2003)

35. Rosier, L.: Homogeneous Lyapunov function for homogeneous continuous vector field. Systems\& Control Lett. 19, 467-473 (1992)

36. Rothschild, L., and Stein, E.: Hypoelliptic differential operators and nilpotent groups. Acta Mathematica 137, 247-320 (1976)

37. Ryan, E.: Universal stabilization of a class of nonlinear systems with homogeneous vector fields. Systems \& Control Lett. 26, 177-184 (1995)

38. Sepulchre, R., and Aeyels, D.: Stabilizability does not imply homogeneous stabilizability for controllable systems. SIAM J. Control Optimization 34(5), 1798-1813 (1996)

39. Teel, A. R.: Connections between Razumikhin-type theorems and the ISS nonlinear small gain theorem. IEEE Trans. Automat. Control 43(7), 960-964 (1998)

40. Zubov, V.: On systems of ordinary differential equations with generalized homogeneous righthand sides. Izvestia vuzov. Mathematica. 1, 80-88 (1958) in Russian. 